\title{
Prognostic impact of typical and probable usual interstitial pneumonia pattern in idiopathic pulmonary fibrosis: is the debate about biopsy a Star Wars saga?
}

\author{
Martin Kolb (1) ${ }^{1}$, Ganesh Raghu² and Athol Wells ${ }^{3}$ \\ Affiliations: ${ }^{1}$ Dept of Respiratory Medicine, Pathology and Molecular Medicine, McMaster University, \\ Hamilton, ON, Canada. ${ }^{2}$ CENTER for Interstitial Lung Disease, University of Washington, Seattle, WA, USA. \\ ${ }^{3}$ Interstitial Lung Disease, Royal Brompton Hospital, Royal Brompton Hospital, London, UK.
}

Correspondence: Martin Kolb, Dept of Respiratory Medicine, Pathology and Molecular Medicine, McMaster University, 50 Charlton Ave East, Hamilton, ON L8N 4A6, Canada. E-mail: kolbmamcmaster.ca

@ERSpublications

The debate about the benefit of performing a surgical lung biopsy in patients with the probable UIP pattern needs every case to be viewed on an individual basis. This editorial attempts to bring some clarity to treating physicians and confused patients. http://bit.ly/2Qk517r

Cite this article as: Kolb M, Raghu G, Wells A. Prognostic impact of typical and probable usual interstitial pneumonia pattern in idiopathic pulmonary fibrosis: is the debate about biopsy a Star Wars saga? Eur Respir J 2020; 55: 2000590 [https://doi.org/10.1183/13993003.00590-2020].

\section{Prelude}

The diagnosis of interstitial lung diseases (ILDs) can be straightforward for some experienced experts without biopsy data, but not so straightforward to others. In the context of suspected idiopathic pulmonary fibrosis (IPF), accurate non-invasive diagnosis requires recognition of the hallmark usual interstitial pneumonia (UIP) pattern on imaging, obviating lung biopsy $[1,2]$. When the imaging pattern is "probable UIP", the need for histopathology confirmation of a UIP pattern in surgical lung biopsy (SLB) is often debated, despite concordance between expert groups that SLB is not mandated for IPF diagnosis when the clinical setting is appropriate and multidisciplinary discussion has taken place [1-3]. It is the radiographic pattern of "probable UIP" that is at the centre of this debate, and the question of whether a biopsy is required in order to diagnose a specific fibrotic ILD. Let us recapitulate the terminology first before summarising new evidence and arguing about the appropriate diagnostic steps. The "typical UIP pattern" on high-resolution computed tomography (HRCT) consists of peripheral reticulation with an apicobasal gradient, traction bronchiectasis and honeycombing. IPF is a definite diagnosis when this pattern is present and no cause for it is identified clinically. That is the easy one. The "probable UIP pattern" on HRCT includes the same descriptors as above, except that honeycombing is absent. This HRCT pattern can be IPF but it also can be something different. Now that sounds more difficult; or is it not?

\section{Episode One}

Patients with IPF who have the typical UIP pattern have been the prototype participants in the clinical trials of the past 15 years [4]. Most of our knowledge about the clinical behaviour of IPF is derived from this group. Nearly $40 \%$ of patients enrolled in the INPULSIS trials were assumed to have IPF despite the lack of honeycombing (i.e. they had a probable UIP pattern and a clinical diagnosis of IPF was made by the enrolling clincians without SLB to confirm a histopathology pattern of UIP). The rate of forced vital capacity 
(FVC) decline was similar in the placebo arm in those with a 2011 guideline-adherent diagnosis of IPF and in those with probable UIP on HRCT and no biopsy confirmation [5]. The treatment response to nintedanib was also strikingly similar in these two subgroups. However, it must be acknowledged that some patients wih probable UIP on HRCT may have had other fibrotic lung diseases, including nonspecific interstitial pneumonia or fibrotic hypersensitivity pneumonitis, with an IPF-like presentation and clinical course and, thus, it cannot be assumed that all patients with the probable UIP pattern have IPF.

The division between clinicians in applying these data to IPF diagnosis is the time-honoured division between dogmatism and empiricism, the great rival medical traditions of ancient Alexandria. Whilst "first do no harm" is the dictum that all ILD clinicians adhere to, the ongoing debate is whether SLB is needed in patients with probable UIP on HRCT following multidisciplinary discussion, in the absence of absolute contraindications and with patient acceptance of the risks associated with SLB. Experts differ, they are perfectly right in doing so. The precept of the dogmatists is that a doctor can understand how to treat a patient only by certain knowledge of "the hidden state": some clinicians accept nothing less than a definite diagnosis. Others argue with the empiricists that the connection between the presentation of a disease and its treatment is a matter of experience: a probable or "working" diagnosis of IPF is sufficient, with SLB performed only when the clinical picture is not classical (the classical profile being older males, often former or current smokers; figure 1).

In guidelines, recommendations tend to be made using terms such as "majority" or "many", and "few" or "exceptional" patients. Thus, decisions on diagnostic investigation and management are deferred to the treating physician, in partnership with the patient, on a case by case basis. This should not be viewed as lack of guidance but, rather, recognition that a "one size fits all approach" is inappropriate in IPF diagnosis. In fact, the guideline recommendation for IPF diagnosis is for the "patient suspected to have IPF" and not for all patients with ILD. Relative contraindications to SLB (age, cormobidities, disease severity), the mortality associated with SLB and the wishes of patients are the key determinants of SLB recommendations in individual cases. Worked into this clinical conundrum is the concept of management based on a working diagnosis of IPF. The recent update of the international IPF guideline can be viewed as statement on data needed to make a definite diagnosis of IPF, including, in many patients, SLB. The guideline is not mandating the need for SLB in patients with probable UIP in the appropriate clinical setting and emphasises that the decision of subjecting the patient to SLB should be handled by multidisciplinary discussion $[2,6]$. The question of whether a working diagnosis of IPF, made with $70-90 \%$ likelihood (implying that approximately $20 \%$ of patients will not, in fact, have IPF) is an acceptable compromise is deferred to the confronted patients and physician. But, there again, the level of confidence is subjective and depends on the experience and wisdom of the expert: "what the mind knows, the eye sees".

\section{Probable UIP}
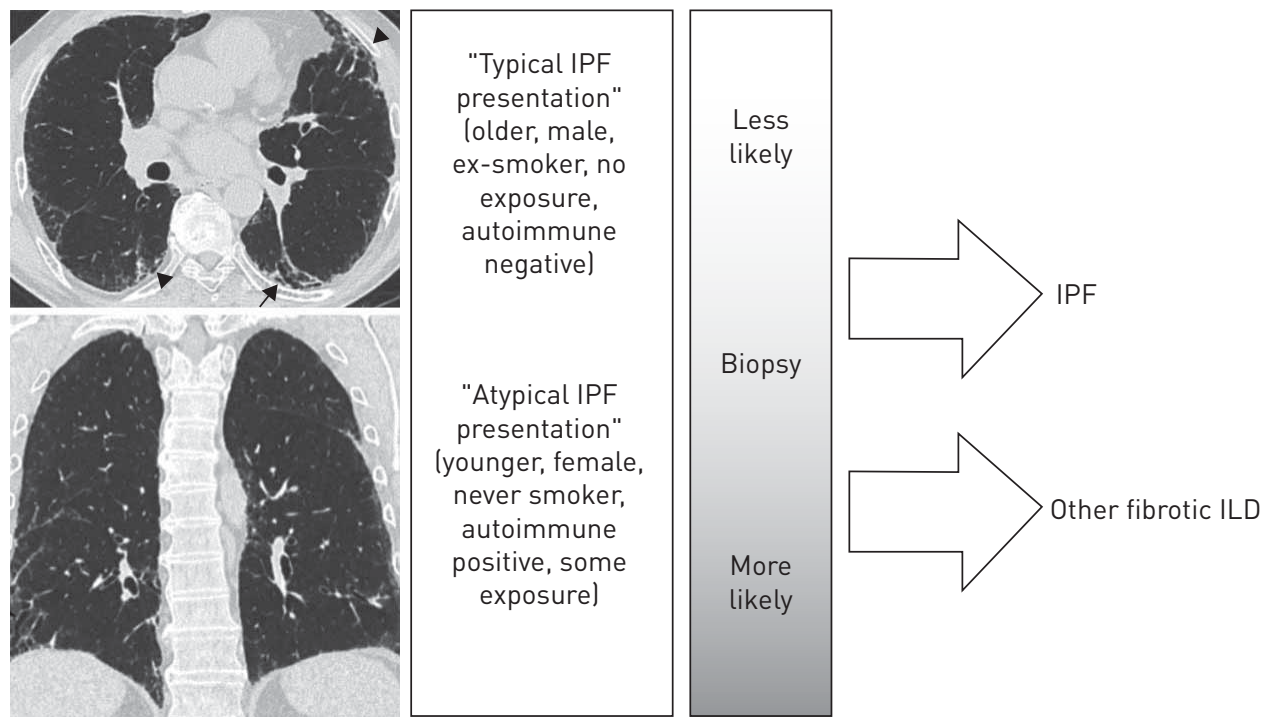

FIGURE 1 The "Star Wars saga" of whether to subject the patient with the "probable UIP" lusual interstitial pneumonial pattern to lung biopsy or not appears to some as a seemingly endless debate. However, if made as an individualised decision between the managing physician and the patient along a smooth scale of idiopathic pulmonary fibrosis (IPF) being more or less likely, it may be straightforward to experts. ILD: interstitial lung disease. Radiology images reproduced from reference [2] with permission from the American Thoracic Society. 


\section{Episode Two}

In a study published in this issue of the European Respiratory Journal, FukIHARA et al. [7] report that patients with a probable UIP pattern on initial HRCT had a better prognosis, survival and longer time to first episode of acute exacerbation than those with a typical UIP pattern. Although $83 \%$ of biopsied subjects with a probable UIP pattern on HRCT had a histopathological pattern of UIP, the diagnosis of IPF was made in only $66 \%$ of patients after multidisciplinary discussion. In this patient subgroup, outcomes were identical to patients with an IPF diagnosis and typical UIP pattern on HRCT.

While the results indicate that an IPF diagnosis is not synonymous with a probable UIP pattern on HRCT, the findings must be interpreted with caution: 1) the study was retrospective and from a single center in Japan with no standardised treatment regimen; and 2) importantly, the multidisciplinary discussion in this study integrated disease behaviour during subsequent follow-up, which was not available at the time of SLB. The quality of these data differs fundamentally from observations in prospective IPF treatment trials, which provide most of the data that inform international guidelines. Nevertheless, the diagnostic and outcome heterogeneity in patients with probable UIP on HRCT in this study is an important reality check. By all means, feel free to make a working diagnosis of IPF on pragmatic grounds... but be prepared for a good long-term outcome, indicative of a diagnosis other than IPF, in some patients and expect to revise the diagnosis based on disease behaviour.

\section{Episode Three}

The recent findings in the INBUILD trial have initiated another debate between lumpers and splitters with regard to the natural history and treated course of the individual fibrosing ILDs, including the idiopathic interstitial pneumonias and fibrosing ILDs with known causes [8, 9]. Some, if not many, have misunderstood the message of INBUILD. In INBUILD, a combined cohort of patients with diagnoses other than IPF and progression of disease despite management, had a decreased rate of decline in FVC with nintedanib treatment when the heterogeneous group of fibrosing ILD with demonstrated progression in lung disease was lumped into a single entity: the PF-ILD (progressive fibrotic ILD) [8]. The treatment effects in this trial were significant in the grouped patients with PF-ILD of different aetiology and this has caused some to argue that ILD diagnosis in general, and the distinction between IPF and other fibrosing diseases in particular, no longer appears to matter as management may no longer differ. It is unkown if the treatment response of the patient with individual fibrotic ILD (e.g. hypersensitivity pneumonitis) to nintedanib is similar to treatment response observed in patients with IPF.

Nintedanib in INBUILD was introduced as second line treatment (or, in some cases, as third or fourth line treatment), with trial enrolment contingent on disease progression despite previous management. Thus, the trial studied an intervention made long after initial diagnosis and management. The view that anti-fibrotic therapy can be indiscriminately instituted when fibrosing lung disease first presents, with diagnosis now irrelevant, disenfranchises a major patient subgroup with a good outcome with initial disease-specific interventions. Initial management strategies other than anti-fibrotic therapy, such as disease modifying immune modulating agents for ILD associated with connective tissue diseases and avoidance of environmental exposures known to induce hypersensitivity pneumonitis may improve outcomes that are meaningful to patients with progressive fibrotic ILDs, other than IPF. Specific diagnosis matters every bit as much, post INBUILD, as it ever did. And INBUILD should not be used as an argument for obtaining biopsy confirmation or not in patients with the probable UIP pattern. This argument is debated at the time of diagnosis and not at the time when one has information about disease progression despite optimum treatment that includes disease modifying, immune modulating agents and avoidance of exposure to environmental factors kown to have contributed to manifested progressive fibrotic ILD.

\section{Final}

Given the ongoing uncertainties in this area, the community clinician and all stakeholders need guidance on the indications for anti-fibrotic therapy in progressive fibrotic lung disorders other than IPF. More observations are required, including further analysis of INBUILD data, parallel analyses of pirfenidone therapy and ongoing work, before management can be addressed in clinical practice guidelines. In the meantime, we argue strongly that existing diagnostic algorithms in ILD should not be modified. In this regard, the findings of FUKIHARA et al. [7] provide strong support for the continued judicious use of biopsy procedures in selected patients with suspected IPF and probable UIP on computed tomography. The Star Wars saga of typical UIP, probable UIP, fibrotic ILD (fILD) and progressive fibrotic ILD (PF-ILD) will certainly keep going for a while. We shall not forget where we have come from just 20 years ago (i.e. steroids and diagnostic apathy for anyone with pulmonary fibrosis) to a sophisticated academic debate and access to disease changing therapies for a substantial number of patients with pulmonary fibrosis nowadays. 
Conflict of interest: M. Kolb reports grants from Canadian Pulmonary Fibrosis Foundation, Canadian Institute for Health Research, Alkermes and Actelion, grants and personal fees for advisory board work from Pulmonary Fibrosis Foundation, Boehringer Ingelheim, Roche Canada and Prometic, personal fees for advisory board work from Gilead, editorial allowance from European Respiratory Journal, and has been site PI in clinical trials for Roche and Boehringer Ingelheim, outside the submitted work. G. Raghu has provided consultancy for Avalyn, BI, Bellerophan, BMS, Biogen, Blade Therapeutics, Fibrogen, Promedior, Nitto, Respivant, Roche-Genentech, Veracyte and Zambon, outside the submitted work. A. Wells reports personal fees for consultancy and lectures from Boehringer Ingelheim and Roche, personal fees for consultancy from Blade, outside the submitted work.

\section{References}

1 Lynch DA, Sverzellati N, Travis WD, et al. Diagnostic criteria for idiopathic pulmonary fibrosis: a Fleischner Society White Paper. Lancet Respir Med 2018; 6: 138-153.

2 Raghu G, Remy-Jardin M, Myers JL, et al. Diagnosis of idiopathic pulmonary fibrosis. An Official ATS/ERS/JRS/ ALAT Clinical Practice Guideline. Am J Respir Crit Care Med 2018; 198: e44-e68.

3 Collins BF, Raghu G. Antifibrotic therapy for fibrotic lung disease beyond idiopathic pulmonary fibrosis. Eur Respir Rev 2019; 28: 190022.

4 Raghu G. Idiopathic pulmonary fibrosis: lessons from clinical trials over the past 25 years. Eur Respir J 2017; 50: 1701209.

5 Raghu G, Wells AU, Nicholson AG, et al. Effect of nintedanib in subgroups of idiopathic pulmonary fibrosis by diagnostic criteria. Am J Respir Crit Care Med 2017; 195: 78-85.

6 Richeldi L, Wilson KC, Raghu G. Diagnosing idiopathic pulmonary fibrosis in 2018: bridging recommendations made by experts serving different societies. Eur Respir J 2018; 52: 1801485.

7 Fukihara J, Kondoh Y, Brown KK, et al. Probable usual interstitial pneumonia pattern on chest CT: is it sufficient for a diagnosis of idiopathic pulmonary fibrosis? Eur Respir J 2020; 55: 1802465.

8 Flaherty KR, Wells AU, Cottin V, et al. Nintedanib in progressive fibrosing interstitial lung diseases. $N$ Engl J Med 2019; 381: 1718-1727.

9 Wells AU, Brown KK, Flaherty KR, et al. What's in a name? That which we call IPF, by any other name would act the same. Eur Respir J 2018; 51: 1800692. 